\title{
Percepção dos pacientes atendidos em Unidades Básicas de Saúde sobre o conhecimento e profilaxia da toxoplasmose
}

\author{
Perception of patients attended in Basic Health Units about toxoplasmosis knowledge and \\ prophylaxis \\ Percepción de los pacientes atendidos en Unidades Básicas de Salud sobre el conocimiento y \\ profilaxis de toxoplasmosis
}

\section{Resumo}

Objetivou-se com este estudo avaliar a percepção de pacientes atendidos nas Unidades Básicas de Saúde (UBS) do município de Patos de Minas, MG, sobre o conhecimento e profilaxia da toxoplasmose. Foi realizado um inquérito observacional transversal prospectivo nos meses de novembro e dezembro de 2019. Aos entrevistados foi aplicado um questionário com 10 perguntas. Dos 2.213 entrevistado 52\% (1.147/2.213) relataram ter conhecimento sobre a toxoplasmose; $49.66 \%$ (1.099/2.213) relataram conhecer as espécies envolvidas na transmissão, desses $89,89 \%$ (988/1099) identificaram o gato, 32,57\% (358/1099) cães, 9,28\% (102/1099) bovinos, 5\% (55/1099) mosquitos e 3,82 \% (42/1099) aves. Quanto a higienização de frutas, verduras e hortaliças, 95,75\% (2119/2.213) realizam o procedimento antes do consumo, e desses 36,33\% (770/2119) utilizam algum produto para desinfecção. Em relação ao consumo de carne $97 \%$ (2163/2.213) consomem o alimento e 76,83\% (1.662/2.163) optam pelo consumo da carne bem assada. A criação de animais foi relata por 65,56\% (1451/2213) dos entrevistados e a criação de gatos por 32,5\% (472/1451). Quanto ao local de defecação dos felinos 44,27\% (209/472) utilizam a caixa de areia e 97\% (203/209) realizam a limpeza da mesma. O principal local de descarte das fezes relatado foi o lixo comum 91,42\% (256/280). O livre acesso à rua foi verificado em 63,9\% (302/472) dos felinos. A investigação permitiu a identificação de importantes fatores de risco que contribuem para a toxoplasmose e a possibilidade de se estabelecer ações de controle, profilaxia e vigilância epidemiológica. Sugere-se priorizar ações de conscientização e educação em saúde voltadas à população.

Palavras-chave: Toxoplasma gondii; Zoonoses; Felinos.

\begin{abstract}
The objective of this study was to evaluate the perception of patients treated at Basic HealthUnits (BHU) in the city of Patos de Minas, MG, regarding the knowledge and prophylaxis of toxoplasmosis. A prospective cross-sectional observational survey was conducted in the months of November and December 2019. Respondents were asked a questionnaire with 10 questions. Of the 2,213 interviewed, 52\% (1,147 / 2,213) reported having knowledge about toxoplasmosis; $49.66 \%(1,099$ / 2,213) reported knowing the species involved in transmission, of which $89.89 \%$ (988/1099) identified the cat, 32.57\% (358/1099) dogs, 9.28\% (102/1099) bovines, 5\% (55/1099) mosquitoes and
\end{abstract}


$3.82 \%$ (42/1099) birds. As for the cleaning of fruits and vegetables, 95.75\% (2119 / 2,213) perform the procedure before consumption, and of those $36.33 \%$ (770/2119) use some product for disinfection. Regarding meat consumption, $97 \%$ (2163 / 2,213) consume the food and $76.83 \%(1,662 / 2,163)$ choose to eat well-roasted meat. Animal breeding was reported by $65.56 \%$ (1451/2213) of the interviewees and cat breeding by $32.5 \%(472 / 1451)$. As for the feline defecation location, $44.27 \%$ (209/472) use the litter box and 97\% (203/209) clean it. The main place for the disposal of reported feces was $91.42 \%$ common waste (256/280). Free access to the street was verified in $63.9 \%$ (302/472) of felines. The investigation allowed the identification of important risk factors that contribute to toxoplasmosis and the possibility of establishing control, prophylaxis and epidemiological surveillance actions. It is suggested to prioritize health awareness and education actions aimed at the population.

Keywords: Toxoplasma gondii; Zoonosis; Cats.

\section{Resumen}

El objetivo de este estudio fue evaluar la percepción de los pacientes atendidos en las Unidades Básicas de Salud (UBS) de la ciudad de Patos de Minas, MG, sobre el conocimiento y profilaxis de la toxoplasmosis. En noviembre y diciembre de 2019 se realizó una encuesta prospectiva observacional transversal. A los encuestados se les entregó un cuestionario con 10 preguntas. De los 2.213 entrevistados, el 52\% (1.147 / 2.213) informó tener conocimientos sobre la toxoplasmosis; $49.66 \%$ (1.099 / 2.213) refirió conocer las especies involucradas en la transmisión, de las cuales $89.89 \%$ (988/1099) identificaron al gato, 32.57\% (358/1099) perros, 9.28\% (102/1099) bovinos, 5\% (55/1099) mosquitos y 3,82\% (42/1099) aves. En cuanto a la limpieza de frutas y verduras, el 95,75\% (2119/2213) realiza el procedimiento antes del consumo, y de estos el 36,33\% (770/2119) utiliza algún producto para desinfectar. En cuanto al consumo de carne, el 97\% (2163/2213) consume el alimento y el 76,83\% (1662/2163) opta por consumir carne bien asada. La cría de animales fue informada por el 65,56\% (1451/2213) de los encuestados y la cría de gatos por el $32,5 \%$ (472/1451). En cuanto al lugar donde defecan los felinos, el 44,27\% (209/472) utiliza el arenero y el $97 \%$ (203/209) lo limpia. El principal lugar de disposición de las heces reportado fue la basura común 91,42\% (256/280). El libre acceso a la calle se verificó en el 63,9\% (302/472) de los gatos. La investigación permitió identificar importantes factores de riesgo que contribuyen a la toxoplasmosis y la posibilidad de establecer acciones de control, profilaxis y vigilancia epidemiológica. Se sugiere priorizar acciones de sensibilización y educación en salud dirigidas a la población.

Palabras clave: Toxoplasma gondii; Zoonosis; Gatos.

\section{Introdução}

Nos últimos anos, mediante a verticalização dos centros urbanos no Brasil, a vida dos homens e também dos animais domésticos que os acompanham, passou por modificações. A solicitação de pets adaptados a ambientes menores e que sejam mais independentes, reduzindo a necessidade de cuidados por parte dos tutores, tem aumentado. Segundo Ottoni et al. (2017), uma das espécies mais distribuídas pelo globo é o gato doméstico (Felis silvestris catus), com isso, o homem tem encontrado nos felinos um perfil compatível com o constante processo de urbanização (Marques et al., 2017). O interesse das pessoas na adoção de animais, principalmente de gatos abandonados tem contribuído para o aumento do número dessa população, sejam domiciliados e semi-domiciliados nas residências (Coelho et al., 2010).

De acordo com o levantamento realizado pelo IBGE (Instituto Brasileiro de Geografia e Estatística) em 2018, em relação à presença de gatos, foi estimado 23,9 milhões de animais no país. A região Nordeste se destacou, apresentando uma proporção de $22,7 \%$ e a população de gatos em domicílios brasileiros foi estimada em 22,1 milhões, o que representa aproximadamente 1,9 gato doméstico por domicílio (IBGE, 2015). Estudos comprovam que a relação das pessoas com os animais de estimação pode resultar em benefícios para o homem, como diminuição da pressão sanguínea e redução na ocorrência de doenças cardíacas, além de outras melhorias fisiológicas, psicológicas e sociais (Lima \& Luna, 2012).

Além da proximidade com os humanos, as diferentes utilizações dos animais de companhia, e sua distribuição, permite que gatos e cães compartilhem com o homem, indesejavelmente, cerca de 60 espécies de parasitos (Anaya et al., 2015). O maior convívio entre esses animais e o homem resulta em maior exposição a agentes com potencialidade zoonótica (Castro, 2005). Segundo Monteiro (2017), tanto os animais de rua quanto os domiciliados podem ser responsáveis pela transmissão de doenças parasitárias, bastando o contato do homem com fezes de animais infectados, ou com solo, fômites, alimento e água contaminados. 
Os felinos domésticos são reservatórios de diversos endoparasitos, como os helmintos gastrintestinais e por parasitarem não somente estes hospedeiros como também o homem (Mundim et al., 2004), possuem fundamental importância em saúde pública (Ngui et al., 2014). Algumas infecções como Larva Migrans Visceral e Larva Migrans Cutânea, causadas pelos agentes Toxocara spp. e Ancylostoma spp. respectivamente, (Monteiro et al., 2016), são exemplos de zoonoses parasitárias transmitidas por gatos (Bowman et al., 2006). Além dessas, a toxoplasmose tem relevância importante dentro das doenças infecto-parasitárias, sendo classificada como cosmopolita, com índices de contaminação de 70 a 95\% (Ministério da Saúde, 2010).

Segundo Hill e Dubey (2002), apenas 1\% dos gatos eliminam em algum momento da vida os oocistos do Toxoplasma gondii, demonstrando que o felino não é o principal transmissor da toxoplasmose, sendo fundamental apenas no ciclo do parasito. A transmissão do protozoário para o homem pode ocorrer pela forma congênita, através de taquizoítos, ou adquirida através da inalação, ingestão oral de cistos teciduais contendo bradizoítos ou pela ingestão de oocistos contendo esporozoítos (Hutchison, 1965). Segundo Forsythe (2013), a maioria das causas de infecção de T. gondii para os humanos é de origem alimentar, sendo maior a prevalência da doença em mulheres pelo fato destas estarem expostas aos cistos da carne crua durante o seu preparo.

Diante dessa realidade e importância da toxoplasmose, o médico veterinário tem a responsabilidade de proporcionar melhores condições ambientais, difusão de informações e orientação à população humana quanto aos princípios básicos de saúde, sobretudo no contexto atual de Saúde Única, que traduz a união indissociável entre a Saúde ambiental, humana e animal (Marques et al., 2017). Considerando a importância das diversas infecções provenientes dos animais domésticos, em especial, o gato para a saúde pública, estudos com a perspectiva deste são necessários para o estabelecimento de medidas adequadas de controle, além de permitir avaliar o risco a que se expõem os seres humanos. Diante disso, objetivou-se com este estudo avaliar a percepção de pacientes atendidos nas Unidades Básicas de Saúde (UBS) do município de Patos de Minas, MG, sobre o conhecimento e profilaxia da toxoplasmose.

\section{Metodologia}

\section{Comitê de ética e pesquisa}

O estudo foi aprovado pelo Comitê de Ética e Pesquisa (CEP) do Centro Universitário de Patos de Minas - UNIPAM, sob o protocolo n. CAAE: 33137420.8 .0000 .5549$.

\section{Local e público alvo}

Foi realizado um inquérito observacional transversal prospectivo, conforme Barbosa (2020), nos meses de novembro e dezembro de 2019, no município de Patos de Minas, Minas Gerais (MG), Brasil. O município está localizado na região do Alto Paranaíba do estado de Minas Gerais, Brasil. Possui uma área de 3.190,187 km² e tem uma população de aproximadamente 152.488 habitantes (IBGE, 2020). Foram entrevistados pacientes e acompanhantes que procuraram por atendimento médico nas 17 Unidades Básicas de Saúde (UBS) do município. A amostragem foi baseada em agrupamento nas 17 UBS e os entrevistados selecionados por conveniência conforme disposição dos inqueridos.

\section{Questionário epidemiológico}

Aos entrevistados foi aplicado um questionário com 10 perguntas fechadas, dicotômicas e objetivas em busca da rapidez, facilidade de aplicação, processo e análise, além de menor risco de parcialidade do entrevistador. Os fatores investigados foram: (1) conhecimento sobre a doença; (2) fontes de infecção da toxoplasmose; (3) métodos de higienização de frutas, verduras e hortaliças; (4) consumo de carne; (5) procedência da carne consumida; (6) presença de animais na 
residência; (7) hábitos dos felinos relacionados ao local de defecação; (8) limpeza da caixa; (9) método de descarte das fezes; e (10) livre acesso à rua. Considerou-se critério de exclusão questionários ilegíveis, incompletos e com resposta binária marcada SIM e NÃO simultaneamente. A aplicação dos questionários foi realizada por acadêmicos do curso de Medicina Veterinária do Centro Universitário de Patos de Minas - UNIPAM.

\section{Análise estatística}

As respostas obtidas no questionário foram tabuladas em planilhas do Microsoft Office Excel 2019. As mesmas foram separadas por UBS, para cada variável investigada foi atribuído um código numérico para identificação das respostas afirmativas e negativas. A análise estatística das variáveis qualitativas categóricas foi efetuada por meio do cálculo das frequências absolutas e relativas, por meio da determinação de percentual simples e construção gráfica.

\section{Conscientização sobre medidas preventivas}

Após a aplicação do questionário epidemiológico, foi realizada a conscientização da comunidade a respeito do que é a toxoplasmose, mecanismos de transmissão e formas de prevenção para população humana e animal. Para isso foi elaborado um material educativo com informações técnicas em concordância com a literatura científica sobre a toxoplasmose.

\section{Resultados e Discussão}

Foram entrevistados 2.917 pacientes e selecionados 2.213 questionários. Dos 2.213 entrevistados 67,35\% (1.487/2.213) eram do sexo feminino e 32.65\% (726/2.213) masculino, com idade variando dos 18 até mais de 60 anos, sendo 25,32\% (559/2.213) com idade entre 18 e 29 anos, 55\% (1.204/2.213) adultos entre 30 e 59 anos e 20,38\% (450/2.213) idosos com idade acima de 60 anos. Os resultados das perguntas 1 a 6 encontram-se dispostos na Figura 1.

Figura 1. Representação gráfica das respostas (1 ao 6), obtidas no questionário epidemiológico aplicado à população em atendimento nas Unidades Básicas de Saúde do município de Patos de Minas MG, 2019.

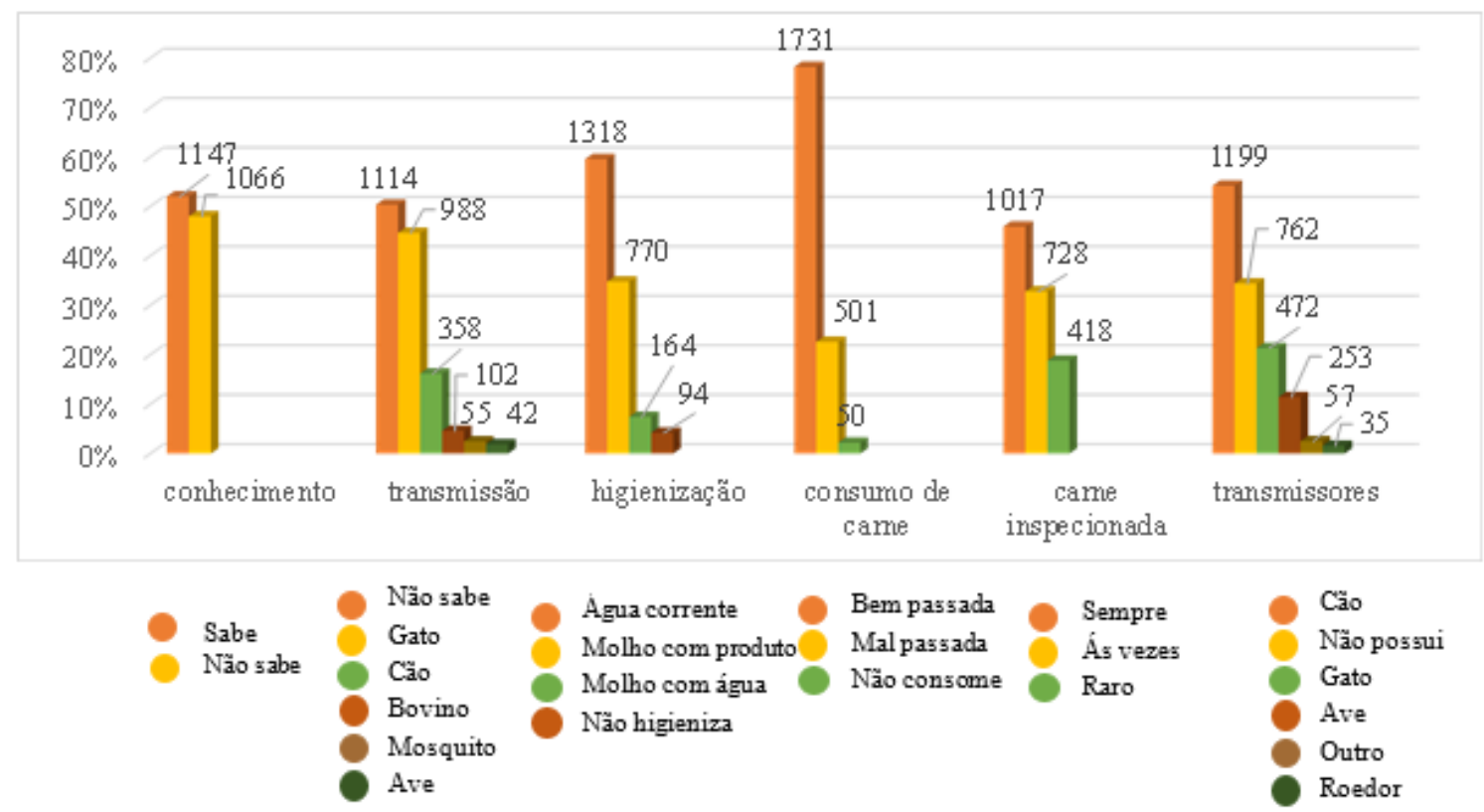

Fonte: Dados da pesquisa dos autores. 
Observou-se que 52\% (1.147/2.213) dos entrevistados relataram ter conhecimento sobre a toxoplasmose. A capacidade de compreender fenômenos relacionados à saúde é um dos pontos mais importantes para a melhoria da qualidade de vida de uma população e para o controle de doenças (Borges et al., 2009). No estudo realizado por Langoni et al. (2006), das 500 gestantes atendidas pela rede pública de saúde da cidade de Niterói - RJ, 54\% nuncatinham ouvido falar sobre toxoplasmose, foi possível observar ainda, que o nível deconhecimento por parte das gestantes aumentou com a idade e com o número de gestações. No estudo de Moura et al. (2019), realizado no Centro de Ciências Biológicas e Saúde com 239 gestantes do município de Imperatriz - MA, somente 23,4\% das gestantes tinham conhecimentos sobre a doença.

Resultados diferentes foram encontrados na pesquisa de Rodrigues (2015), realizada com mulheres na faixa etária classificada como reprodutiva (18 a 49 anos), em Unidades Básicas de Saúde de seis bairros do município de Mossoró-RN, verificou-se que $83 \%$ das mulheres não tinham conhecimento sobre toxoplasmose e suas formas de transmissão. No estudo de Rodrigues (2015), foi analisada a associação do conhecimento sobre a doença com o nível de escolaridade da mulheres entrevistadas, constatou-se que $19,5 \%$ das mulheres com conhecimento sobre a toxoplasmose, possuíam ensino médio completo à pós graduação e daquelas que nunca frequentaram a escola ou tinham o ensino médio incompleto, apenas 1,3\% relataram ter algum conhecimento sobre a toxoplasmose.

Em relação ao fator idade, verificou-se que daqueles que relataram ter conhecimento sobre a doença, 31,39\% (360/1147) tinham idade entre 18 e 29 anos, 56,66\% (650/1147) com idade entre 30 e 59 e 11,94\% (137/1147) com idade acima de 60 anos. No estudo realizado por Moura et al. (2016), avaliou-se o conhecimento da toxoplasmose em gestantes com idade entre 13 a 43 anos, com nível de escolaridade do analfabetismo ao ensino superior, os autores observaram que 57,3\% das entrevistadas desconheciam o termotoxoplasmose e que fatores como aumento da idade e escolaridade podem ter influência na aquisição de conhecimentos sobre a infecção e tornar a adoção e aplicação de medidas preventivas mais eficazes.

Outros estudos como o realizado pelo Instituto Oswaldo Cruz, com gestantes da rede pública de saúde do município de Niterói, Rio de Janeiro, também identificaram que elementos como aumento da escolaridade e idade podem influenciar na aquisição de conhecimento sobre a toxoplasmose. Das gestantes entrevistadas que possuíam o ensino fundamental incompleto apenas $25,6 \%$ conheciam o termo toxoplasmose e das gestantes entrevistadas com ensino superior completo $70 \%$ conheciam o termo toxoplasmose, das gestantes de 13 a 20 anos $26,4 \%$ tinham conhecimento sobre a doença e das gestantes de 31 a 43 anos o percentual subiu para 61,1\% (Moura et al., 2016).

Quanto ao conhecimento das espécies animais capazes de transmitir a doença ao homem, 49.66\% (1.099/2.213) relataram conhecer esses animais, desses 89,89\% (988/1099) identificaram o gato, 32,57\% (358/1099) cães, 9,28\% (102/1099) bovinos, 5\% (55/1099) mosquitos e 3,82 \% (42/1099) aves. Sabe-se que T. gondii infecta naturalmente aproximadamente 200 espécies de mamíferos (humanos, animais domésticos, animais de produção e selvagens), além das aves (Silva et al., 2006), embora o gato seja consideradoo único hospedeiro definitivo urbano, uma vez que somente nele ocorre o ciclo sexuado do parasito (Bonametti et al., 1997), a principal fonte de infecção para os humanos são os animais de produção, além dos felídeos. É importante ressaltar que a infecção por contato direto com gatos excretando oocistos é extremamente improvável, pois os oocistos devem esporular para se tornarem infectantes, desse modo é pouco provável a infecção em decorrência do contato com fezes frescas, assim como é mínima a possibilidade de transmissão para os seres humanos pelo ato de tocar ou acariciar um gato (Camillo, 2015). No estudo de Cook et al., (2000) realizado com mulheres grávidas em diversas regiões da Europa, identificou-se que o contato com felinos não apresentou ser umfator de risco para a infecção por T. gondii.

No estudo realizado por Langoni et al. (2006), a maioria (80\%) dos professores do ensino médio entrevistados, citaram o cão como transmissor da doença. Os cães são considerados um risco potencial para a transmissão do T.gondii, 
pois podem mecanicamente transmitir oocistos para as pessoas e, ainda, em certas partes do mundo, a sua carne é consumida pelo homem (Dubey et al., 2007). Em outro estudo realizado por Barbosa (2020), foi avaliada a percepção de profissionais de saúde sobre toxoplasmose no município de Areia - Paraíba, que ao serem questionadas sobre a participação dos gatos no ciclo da doença, $60 \%$ responderam que eles eram hospedeiros definitivos e $40 \%$ responderam que eles eram hospedeiros intermediários.

Em relação a higienização de frutas, verduras e hortaliças, verificou-se que 95,75\% dos entrevistados (2119/2.213) realizam o procedimento antes do consumo, e desses 36,33\% (770/2119) utilizam algum produto para desinfecção. De acordo com Monteiro et al. (2012), uma das formas de prevenção à toxoplasmose é a adoção de hábitos de higienização de frutas, legumes e verduras, pois estes diminuem as chances de ingestão de alimentos contaminados com oocitos.

A Agência Nacional de Vigilância Sanitária (ANVISA) recomenda que a higienização de hortaliças seja feita com água corrente e com a imersão dos alimentos em solução de hipoclorito de sódio (Ministério da Saúde, 2013). A falta de higienizaçãoe o consumo de hortaliças cruas podem aumentar significativamente as chances de infecçãopelo protozoário T. goondii, como tem sido demonstrado em várias regiões do mundo, inclusive em países como a China (Branco et al., 2012). O referido autor fez uma pesquisacom 499 gestantes e constatou que a maioria $(93,59 \%)$ tem o hábito de consumir vegetais crus. A lavagem mecânica dos vegetais para remoção dos oocistos deve ser uma orientação importante a ser repassada às gestantes, no entanto, esta medida foi relatada por apenas 4,01\% delas.

Resultados diferentes foram encontrados na pesquisa de Batista (2014), realizada com 48 gestantes que faziam acompanhamento pré-natal no Centro de Saúde de Planaltina - DF, onde todas afirmaram lavar as frutas e verduras cruas, $42 \%$ disseram lavar apenas comágua, $21 \%$ disseram fazer a higienização com água e sabão, 15\% com água sanitária, 12\% com água e vinagre, $8 \%$ higienização de forma alternada e $2 \%$ disseram utilizar outros produtos.

Nota-se que a maioria dos entrevistados 76,83\% (1.662/2.163) que consomem carne, optam pelo consumo do alimento bem assado, mas chama a atenção que $23,16 \%$ (501/2.163) realiza o consumo de carnes mal passadas. Pereira et al. (2010) e Bahia et al. (2003), relatam que o modo de infecção mais comum em humanos é por via oral, por ingestão de carne (de animais de produção) crua ou mal cozida, contendo cistos do protozoário, ou de água e frutas ou vegetais crus, contaminados com oocistos de T. gondii.

Segundo Frenkel (2002), carnes originárias das espécies caprina, suína e ovina, contaminadas com oocitos, apresentam uma importante via de transmissão da doença para os humanos; outro estudos realizados por Dubey et al. (1998), nos Estados Unidos, Austrália e Europa apontam que os cistos teciduais em carne bovina aparentam ter menor viabilidadee por esse motivo não foram isolados. De acordo com Cook et al. (2000), o tipo de carne que oferece maior risco para a população varia de acordo com a região. Nesse sentido, é de extrema importância o conhecimento da população sobre a via de transmissão por meio de consumo de carnes cruas ou mal cozidas, de todas as espécies, pois é epidemiologicamente significativa para a diminuição dos índices de infecção (Rodrigues, 2015).

Os resultados dos questionários das perguntas 7 a 10, correspondente aos indivíduos que possuem gatos em casa, encontram-se dispostos na Figura 2. 
Figura 2. Representação gráfica das respostas binárias (7 ao 10) obtidas no questionário epidemiológico sobre a toxoplasmose, aplicados à população em atendimento nas Unidades Básicas de Saúde do município de Patos de Minas MG, 2019.

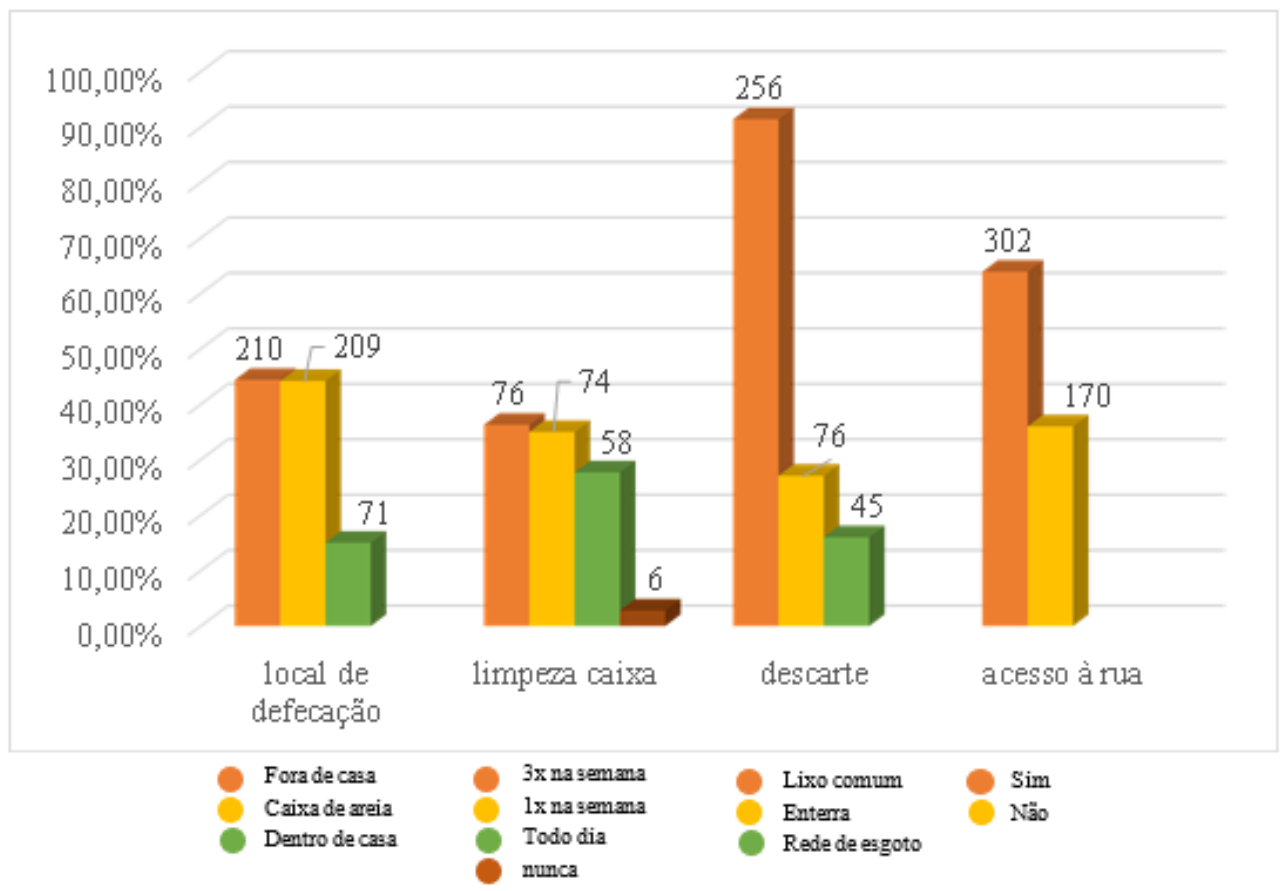

Fonte: Dados da pesquisa dos autores.

A criação de animais foi relatada por 65,56\% (1.451/2.213) dos entrevistados, desses 32,5\% (472/1451) disseram possuir gatos e 44,27\% (209/472) mencionaram a caixa de areia como local utilizado para defecação. Resultados diferentes foram obtidos na pesquisa de Viol et al. (2014), em que do total de entrevistados que possuíam gatos, 74,57\% relataram que os animais defecavam no quintal, 4,3\% dentro de casa/apartamento, e apenas 3,5\% em caixa de areia apropriada. É importante treinar os gatos filhotes a defecarem em caixas com papel absorvente ou areia, removendo os dejetos o mais frequentemente possível, eliminando-os no vaso sanitário do banheiro ou colocando-os no lixo muito bem acondicionados. Embora os oocistos sejam eliminados no ambiente somente pelas fezes dos felídeos recém infectados, a partir de 7 dias pós infecção permanecendo por até 21 dias, no ambiente condições favoráveis de umidade e temperatura propiciam a esporulação dos oocistos, e permitem que os mesmos tornem-se infectantes por anos (Megid et al., 2016).

Apesar da pesquisa apontar que apenas uma pequena parte dos entrevistados 2,87\%(6/209) não realizam a limpeza das caixas de areia, esse percentual merece atenção, pois as fezes contaminadas pelo T. gondii deixadas expostas ao meio ambiente possuem alta probabilidade de se tornarem fontes de contaminação, tendo em vista que os oocitos são altamente resistentes às condições ambientais. No estudo realizado por Moura (2019), com 239 gestantes, no município de Imperatriz -MA, 61,4\% das entrevistadas afirmaram não realizar a limpeza da caixa de areia e das que faziam a limpeza, nenhuma utilizava luvas. Éimportante ressaltar que o problema não é o contato direto dos oocistos com a mão, mas o descuido em levar a mão contaminada aos olhos e à boca. Vescovi et al. (2009), afirmam que a limpeza diária da caixa de areia dos gatos é considerada uma forma eficaz de prevenção à toxoplasmose, pois evita a esporulação do oocisto caso este esteja presente nas fezes.

O descarte das fezes em lixo comum 91,42\% (256/280) e rede de esgoto 16,07\% (45/280) foi relatado pelos entrevistados, sendo as duas opções recomendadas, mas preferivelmente o descarte no vaso sanitário para que ocorra à 
destinação para a estação de tratamento de esgoto (Ortiz \& Leite, 2010). Parte dos entrevistados 27,1\% (76/280) disseram utilizar o enterro das fezes como uma prática de descarte. Segundo a Companhia de Saneamento Básico do Estado de São Paulo - SABESP (2016) as fezes dos gatos são até três vezes mais poluentes do que a de humanos e por esse motivo enterrá-las não é considerada uma prática adequada de descarte. Um hábito importante e que carece de atenção é o não descarte das fezes dos animais, que produz situações de risco e facilita a transmissão de doenças (Etheredge et al., 2004). De acordo com Côrtes et al., (1988) as fezes dos gatos são um problema de saúde pública e por esse motivo, após a limpeza da caixade areia ou de qualquer outro local de defecação, elas devem ser descartadas de forma correta, para que haja menor risco de contaminação de toxoplasmose e de outras zoonoses.

Em um estudo realizado por Côrtes et al., (1988) com 100 moradores da cidade deIlha Solteira - SP identificou-se que a maior parte dos entrevistados desconheciam os mecanismos adequados de descarte dos dejetos dos animais, no entanto $14 \%$ manifestaram ter conhecimento de que o vaso sanitário é uma alternativa para a disposição das fezes. Desses entrevistados $93 \%$ disseram fazer o descarte no lixo comum ou no vaso sanitário e apenas $1 \%$ afirmaram deixar na grama ou no quintal e 6\% na rua. Já a pesquisa de Rodrigues(2015), identificou que 72,80\% faz o descarte no lixo comum e que 27,7\% afirmaram não possuir preocupação com o recolhimento das fezes. Nesse sentido, nota-se a importância de programas de conscientização e educação em saúde dos indivíduos que possuem animais deestimação, principalmente em relação ao local de defecação, frequência da remoção e descarte final, pois o destino incorreto, pode facilitar a transmissão da doença (Ortiz \& Leite, 2010).

Um alto percentual 63,9\% (302/472) de tutores relataram que seus animais possuem livre acesso às ruas. Pinto et al. (2009), evidenciam a importância do ambiente em relação àinfecção pelo protozoário T. gondii. Apesar de gatos vagando pelas ruas ser considerado uma paisagem natural para o homem (Genaro, 2010), impedir o acesso irrestrito dos animais à rua é uma das formas mais importantes para a manutenção da saúde e do bem estar dos felinos (Osório, 2011).

Lucas et al. (1999) e Pena et al. (2006) enfatizam que o livre acesso à rua é um dos fatores de interferência na soroprevalência de $T$. gondii em gatos, devido a oportunidade que os animais encontram nas ruas de caçar pequenas presas e assim se tornarem mais suscetíveis à infecção pelo parasita, do que os animais domiciliados e sem acesso à rua. Moura et al. (2007), afirmam que o meio ambiente contaminado com os oocitos de T. gondii apresenta grande importância sobre a disseminação da toxoplasmose, podendo infectar animais e humanos que o frequentam. Em Porto Alegre - RS, amostras de soro de 245 felinos foram testados pelas técnicas sorológicas de Hemaglutinação Indireta (HAI) e Imunofluorescência Indireta (IFI), de acordo com os resultados obtidos, Pinto et al. (2009) constatou-se que o acesso de gatos à rua contribuiu para a soropositividade desses animais, onde $47,11 \%$ dos que tinham acesso às ruas testaram positivos, um valor elevado quando comparado àqueles que não tinham acesso $(13,5 \%)$.

Após a aplicação dos questionários, realizou-se a conscientização dos entrevistados, sobre os mecanismos de transmissão da doença e medidas de profilaxia para homens e animais. Acredita-se que a divulgação dessas informações possa contribuir para prevenção da doença, e sugere-se que treinamentos contínuos às equipes de saúde sejam realizados, uma vez que esses profissionais estão frequentemente em contato com a população. No estudo realizado por Branco et al. (2012), com profissionais da saúde do município de Maringá sobre conhecimentos básicos e formas de transmissão da toxoplasmose, identificou-se que a maioria dos profissionais não souberam responder aos questionamentos.Fato preocupante para a saúde humana e animal, pois esses profissionais são os responsáveis pela disseminação do conhecimento para a população, evidenciando-se então, a necessidadede capacitação de profissionais da área da saúde sobre essa parasitose.

Na pesquisa realizada por Moura et al. (2018), identificou-se a falta de conhecimento de gestantes em relação as formas de transmissão da toxoplasmose, $85 \%$ declararam não receber nenhum tipo de informação e orientação sobre a parasitose durante as consultas de pré-natal. A falta de informações dos profissionais de saúde para a população,relacionada 
com a falta de conhecimento da população sobre as principais formas de transmissão da doença, torna a compreensão das principais vias de transmissão um desafio para a criação de práticas sanitárias eficientes à prevenção da doença (Kolbekova et al., 2007).

\section{Conclusão}

O inquérito epidemiológico demonstrou que mesmo boa parte dos entrevistados relatarem não ter conhecimento sobre a doença, medidas gerais de profilaxia são realizadas pela população. A investigação permitiu a identificação de importantes fatores de risco (consumo de carne mal passada e livre acesso de gatos à rua), que contribuem para a toxoplasmose e com isso a possibilidade de se estabelecer ações de controle, profilaxia e vigilância epidemiológica. Sugere-se priorizar ações de conscientização e de educação em saúde voltadas à população, além de estudos futuros para maior elucidação da epidemiologia da doença.

\section{Referências}

Anaya, A. M. D.; Medellín, M. O. P. \& Forero, G. J. C. (2015). Nematodes with zoonotic potential in parks of the city of Tunja, Colombia. Salud Publica de México, 57 (2), 170- 176.

Bahia-Oliveira, L. M.; Jones J. L.; Azevedo-Silva, J.; Alves, C. C.; Oréfice, F. \& Addiss D. G. (2003). Highly endemic, waterborne toxoplasmosis in north Rio de Janeiro state, Brazil. Emerging Infectious Diseases, 9 (1), 55-62.

Barbosa, M. J. S. (2020). Percepção dos profissionais de saúde da Atenção Básica sobre toxoplasmose no município de Areia-Paraíba. Monografia. Universidade Federal da Paraíba - Areia, Paraíba, Brasil.

Batista, T. F. R. (2014). Prevalência de fatores de risco à toxoplasmose em gestantes. Monografia. Centro Universitário de Brasília, Brasília, Distrito Federal, Brasil.

Bonametti, A. M., Passos, J. D. N., Silva, E. M. K. D. \& Bortoliero, A. L. (1997). Surto de toxoplasmose aguda transmitida através da ingestão de carne crua de gado ovino. Revista da Sociedade Brasileira de Medicina Tropical, 30 (1), 21-25.

Borges, T. T., Rombaldi, A. J., Knuth, A. G. \& Hallal, P. C. (2009). Conhecimento sobre fatores de risco para doenças crônicas: estudo de base populacional. Cadernos de Saúde Pública, 25 (7), 1511-1520.

Bowman, D. D., Lynn, R. C., Eberhard, M. L. \& Alcaraz, (2006) Parasitologia veterinária de Georgis. (8a ed.), Manole.

Branco, B. H. M., Araújo, S. M. \& Falavigna-Guilherme, A. L. (2012). Prevenção primária da toxoplasmose: conhecimento e atitudes de profissionais de saúde e gestantes do serviço público de Maringá, estado do Paraná. Scientia Medica, 22 (4), 185-190.

Camillo, G. (2015). T. gondii em galinhas domésticas: epidemiologia, isolamento e caracterização molecular. Tese, Programa de Pós-Graduação em Medicina Veterinária, Universidade Federal de Santa Maria, Santa Maria, Rio Grande do Sul, Brasil.

Castro, J. M., Santos, S. V. \& Monteiro, N. A. (2005). Contaminação de canteiros da orla marítima do Município de Praia Grande, São Paulo, por ovos de Ancylostoma e Toxocara em fezes de cães. Revista da Sociedade Brasileira de Medicina Tropical, 38 (2), 199 - 201.

Coelho, W. M. D., Amarante, A. F. T., Soutello, R. V. G., Meireles, M. V. \& Bresciani, K. D. S. (2010). Detecção de helmintos e protozoários de importância em saúde pública em amostras fecais de gatos no município de Andradina, São Paulo. Veterinária e Zootecnia, Botucatu, 17 (1), $95-100$.

Cook, A. J., Gilbert, R. E., Buffolano, W. Z. J., Petersen, E., Jenum, P.A., Foulon, W., Semprini, A. E. \& Dunn, D. T. (2000). Sources of Toxoplasma infection in pregnant women: a European multicentre case-control study. British Medical Journal, Edinburgh, 321 (7254), $142-147$.

Côrtes, V. A., Paim, G.V. \& Filho, R. A. A. (1988). Infestação por Ancilostomídeos e toxocarídeos em cães e gatos apreendidos em vias públicas, São Paulo (Brasil). Revista de Saúde Pública, 22 (4), 341-343.

Dubey, J. P., Lam, T. T. H., Sundar, N. \& Su, C. (2007). Geneticcharacterization of Toxoplasma gondii isolates in dogs from Vietnam suggests their South American origin. Veterinary Parasitology, 146 (3-4), 347-351.

Dubey, J. P., Lindsay, D. S. \& Speer, C. A. (1988). Structures of Toxoplasma gondii tachyzoites, bradysoites and spororozoites and biology and development of tissue cysts. Clinical Microbiology Reviews, 11 (2), 267-299.

Etheredge, G. D., Michael, G., Muehlenbein, M. P. \& Frenkel, J. K. (2004). The roles of cats and dogs in the transmission of Toxoplasma infection in Kuna and Embera children in eastern Panamá. Pan American Journal of Public Health, 16 (3), 176-86.

Frenkel, J.K. Toxoplasmose. In: Veronesi R, Focaccia R, eds. (2002). Tratado de Infectologia. Guanabara Koogan.

Genaro, G. (2010). Gato doméstico: futuro desafio para controle da raiva em áreas urbanas. Pesquisa Veterinária Brasileira, 30 (2), 186-189. 
Hill, D. E. \& Dubey, J. P. (2002). Toxoplasma gondii: transmission, diagnosis and prevention. Clinical Microbiology and Infection, 8 (10), 634-640.

Hutchison, W. M.(1965). Experirmental transmission of Toxoplasma gondii. Nature London, 206 (4987), 961-962.

IBGE - Instituto Brasileiro de Geografia e Estatística. (2015). Pesquisa nacional de saúde. IBGE, 2015.

IBGE - Instituto Brasileiro de Geografia e Estatística. (2018). Censo Pet: População de animais de estimação em todo o território nacional.

IBGE - Instituto Brasileiro de Geografia e Estatística. (2020). Pesquisa Nacional por Amostra de Domicílios: síntese de indicadores. Patos de Minas, 2020.

Kolbekova, P., Kourbatova, E., Novotna, M., Kodym, P. \& Flegr, J. (2007). New and old risk-factors for Toxoplasma gondii infection: prospective crosssectional study among military personnel in the Czech Republic. Clinical Microbiology and Infection, 13 (10), $1012-1019$.

Lucas, S. R. R., Hagiwara, M. K., Loureiro, V. D. S., Ikesaki, J. Y. H. \& Birgel, E. H. (1999). Toxoplasma gondii infection in Brazilian domestic outpatient cats. Revista do Instituto de Medicina Tropical de São Paulo, 41 (4), 221-224.

Langoni, H. (2006). Doenças ocupacionais em avicultura. In: Andreatti Filho, R. L. Saúde aviária e doenças. Roca.

Lima, A. F. M. \& Luna, S. P. L. (2012). Algumas causas e consequências da superpopulação canina e felina: acaso ou descaso? Revista de Educação Continuada em Medicina Veterinária e Zootecnia, 10 (1), 32-38.

Marques, S. M. T., Oliveira, M. R. F. \& Gomes, M. J. T. de M. (2017). Parasitos gastrintestinais em gatos da cidade de Porto Alegre, Rio Grande do Sul. Pubvet Medicina Veterinária e Zootecnia, 11 (11), 1132-1137.

Ministério da Saúde.(2010). Secretaria de Vigilância em Saúde. Doenças infecciosas e parasitárias: Guia de bolso. (8a ed.).

Ministério da Saúde. (2013). Agência Nacional de Vigilância Sanitária. Higienize as mãos: salve vidas. Portal da ANVISA, Brasília.

Monteiro, M. F. M., Ramos, R.A.N, Calado, A.M.C., Lima, V.F.S., Ramos, I.C.N., Tenório, R.F.L., Faustino, M.A.G. \& Alves, L.C. (2016). Gastrointestinal parasites of cats in Brazil: frequency and zoonotic risk. Revista Brasileira de Parasitologia Veterinária, 25 (2), $254-257$.

Monteiro, S. G. (2017). Parasitologia na Medicina Veterinária. Roca.

Moura, F. L. D., Goulart, P. R. M., Moura, A. P. P. D., Souza, T. S. D., Fonseca, A. B. M. \& Amendoeira, M. R. R. (2016). Fatores associados ao conhecimento sobre a toxoplasmose entre gestantes atendidas na rede pública de saúde do município de Niterói, Rio de Janeiro, 2013-2015. Epidemiologia e Serviços de Saúde, 25 (3), 655- 661

Moura, D. S., Oliveira, R. C. M. \& Matos-Rocha, T. J. (2018). Toxoplasmose gestacional: perfil epidemiológico e conhecimentos das gestantes atendidas na unidade básica de saúde de um município alagoano. Arquivos médicos dos Hospitais e da Faculdade de Ciências Médicas da Santa Casa de São Paulo, 63 (2), 69-76.

Moura, I. P. D. S., Ferreira, I. P., Pontes, A. N. \& Bichara, C. N. C. (2019). Conhecimento e comportamento preventivo de gestantes sobre Toxoplasmose no município de Imperatriz, Maranhão, Brasil. Ciência \& Saúde Coletiva, 24 (10), 3933- 3946.

Mundim, T. C. D., Oliveira Júnior, S. D., Rodrigues, D. C. \& Cury, M. C. (2004). Frequência de helmintos em gatos de Uberlândia, Minas Gerais. Arquivo Brasileiro de Medicina Veterinária e Zootecnia, 56 (4), 562-563.

Ngui, R., Lim, Y. A., Ismail, W. H. W., Lim, K. N. \& Mahmud, R.(2014). Case report: zoonotic Ancylostoma ceylanicum infection detected by endoscopy. American Journal of Tropical Medicine, 91 (1), 86-88.

Osório, A. (2011). Posse responsável: moral, ciência e educação ambiental em um grupo de protetores de gatos de rua. Revista de Antropologia Urbana, 3 (2), $51-75$.

Ottoni, C., Van Neer, W., De Cupere, B., Daligault, J., Guimaraes, S., Peters, J. \& Bălăşescu, A. (2017). The palaeogenetics of cat dispersal in the ancient world. Nature Ecology and Evolution, 1, 0139.

Pereira, K. S., Franco, R. M. B. \& Leal, D. A. G. (2010). Transmission of toxoplasmosis (Toxoplasma gondii) by foods. Advances in Food and Nutrition Research, 60, 1- 19

Pinto, L. D., Araujo, F. A. P. D., Stobb, N. S. \& Marques, S. M. T. (2009). Soroepidemiologia de Toxoplasma gondii em gatos domiciliados atendidos em clínicas particulares de Porto Alegre, RS, Brasil. Ciência Rural, 39 (8), 2464-2469.

Rodrigues, D. N. J. (2015). Avaliação do conhecimento da população sobre formas de transmissão e medidas preventivas da toxoplasmose em Mossoró - RN. Dissertação, Universidade Federal Rural do Semi-Árido, Mossoró, Rio Grande do Norte, Brasil.

Silva, F. W. S., Alves, N. D., Amóra, S. S. A., Texeira, F. H. V., Accioly, M.P., Carvalho, C. G., Nóbrega, R. M., Filgueira, K. D. \& Feijó, F. M. C. (2006). Toxoplasmose: uma revisão. Revista Ciência Animal. 16 (2), $71-77$.

Viol, M. A., Matos, L. V. S., Aquino, M. C. C. D., Alves, I. P., Bregadioli, T., Oliveira, B. C. M. \& Bresciani, K. D. S. (2014). Avaliação do grau de conhecimento sobre leishmaniose e toxoplasmose em moradores do município de Araçatuba, SP. Veterinária e Zootecnia, 2 (2), $306-313$. 